\title{
Asteroseismic Analysis of Pulsating Subdwarf B Stars
}

\section{S. Charpinet ${ }^{1}$}

\author{
LAT, Observatoire Midi-Pyrénées, Toulouse, France \\ G. Fontaine, P. Brassard \\ Département de Physique, Université de Montréal, Québec, Canada
}

\begin{abstract}
We briefly present a method to perform detailed asteroseismological analyses of pulsating subdwarf B (sdB) stars (also referred to as the EC14026 stars). We use a forward approach based on the computation of large grids of sdB stellar models (each model being analyzed with a linear nonadiabatic pulsation code) to derive, in an objective way, the most appropriate set of model parameters that can best reproduce the observed period spectrum of a given $\mathrm{sdB}$ pulsator. We discuss an illustrative application of this method to the pulsating sdB star PG 1047+003, for which we derive the fundamental parameters $T_{\text {eff }}, \log g, M_{\text {tot }}$, and $M_{\text {env }}$.
\end{abstract}

\section{Introduction}

Pulsating sdB stars (also known as the EC14026 stars, the prototype of the class whose discovery was reported in 1997; Kilkenny et al., 1997) are hot, compact objects that exhibit rapid multiperiodic $(P \sim 80-600 \mathrm{~s}$ ) low amplitude (a few millimag) luminosity variations attributed to low degree, low order $p$-modes (see, for example, the review of Kilkenny in these proceedings). Subdwarf B stars are identified to the so-called Extreme Horizontal Branch (EHB) stellar models, and thus are evolved, low mass $\left(M \sim 0.5 M_{\odot}\right)$ core helium burning objects (Dorman et al., 1993).

The theory of pulsating sdB stars, whose origin is anterior to their observational discovery (see Charpinet et al., 1996), has now reached a relatively mature state. The mode driving mechanism identified is a $\kappa$-effect involving the $Z$-bump which becomes efficient because heavy metals (especially iron, the main contributor to the $Z$-bump) can accumulate locally into abundances much higher than solar in the envelope of these stars due to microscopic diffusion processes (gravitational settling and radiative levitation). Nonadiabatic pulsation computations using stellar models that include the nonuniform equilibrium abundance profiles of iron derived from diffusion calculations have given results remarkably consistent (both qualitatively and quantitatively) with the data gathered so far (see

\footnotetext{
${ }^{1}$ Postdoctoral Fellow, funded by the CNES, France; e-mail: scharpin@ast.obs-mip.fr
} 
the review of Charpinet et al., 2001). In this context, detailed asteroseismological analyses of sdB pulsators should now become possible.

We briefly describe in the next section (Section 2) the method developed to perform the detailed asteroseismic analysis of pulsating sdB stars. In Section 3, illustrative results obtained when applying this approach to the sdB pulsator PG 1047+003 are presented and discussed. A similar result has been obtained for another sdB pulsator (PG 0014+067; see Brassard et al., 2001).

\section{Description of the method}

Our goal is to objectively derive the set of model parameters that best reproduce the observed pulsation properties of a given EC14026 star. Adopting a forward approach, we build large grids of sdB star models to explore the parameter space for which at each node (defined by the specified model parameters) we compute:

1 - The stellar structure corresponding to the 4 model parameters: the effective temperature $\left(T_{\text {eff }}\right)$, the surface gravity $(\log g)$, the total mass of the star $\left(M_{\text {tot }}\right)$, and the quantity $\log q(H) \simeq \log \left(M_{\text {env }} / M_{\text {tot }}\right)$ related to the mass of the H-rich envelope $\left(M_{\text {env }}\right)$. These models are our so-called 2 nd generation models which include the equilibrium nonuniform iron abundance profiles expected from microscopic diffusion (see Charpinet et al., 2001).

2 - The nonadiabatic pulsation quantities (periods and stability information) of the stellar model calculated previously within a specified period window (defined by the range of observed periods of the star being analyzed).

3 - The best match of the observed periods to the computed periods. This last step is performed using a Genetic Algorithm (GA) based optimization code which ensures that the best combination of $N$ observed periods ( $\left.P^{\text {obs }}\right)$ among a set of $T(T>N)$ theoretical periods $\left(P^{\text {theo }}\right)$ is found. The following merit function is minimized to find the best solution:

$$
\chi^{2}=\sum_{i=1}^{N}\left(\frac{P_{i}^{\text {obs }}-P_{i}^{\text {theo }}}{\sigma_{i}}\right)^{2}
$$

The period-matching process is done without any initial assumption (or constraint) as of the individual values of $\ell$ and $k$ of the observed periods.

Due to the large number of models that needs to be computed to build such 4Dimensional grids with sufficient resolution and parameter space coverage, the whole computation process is parallelized, using the local network as a computer cluster.

The result of this grid computation is the quantity $\chi^{2}$ as a function of the 4 fundamental parameters $T_{\text {eff }}, \log g, M_{\text {tot }}$, and $\log q(H)$, as well as the detailed period fit (i.e., the mode identification) at each node of the grid. Our last step is then to search for the deepest minima in the $4 \mathrm{D}-\chi^{2}$ hypersurface which correspond to possible solutions. Tests with artificial data show that we are able to retrieve the right solution (in the model parameter space) and mode identification this way. We stress that with this method, the mode identification comes as a byproduct of the asteroseismic analysis and is not an initial assumption. 


\section{Application to PG $1047+003$}

We applied this method to the sdB pulsator PG $1047+003$ by attempting to fit the observed periods. This object was chosen because excellent data sets of white light fast-photometry were obtained both from the Canada-FranceHawaii $3.6 \mathrm{~m}$ telescope $(\sim 10 \mathrm{~h}$ gathered with LAPOUNE in 1998) and from a dedicated multisite campaign ( $\sim 98 \mathrm{~h}$ with a $\sim 30 \%$ duty cycle; Kilkenny et al., in preparation). Preliminary analysis of the light curves revealed 19 independent modes (excluding splitted components) with periods in the range $90-175 \mathrm{~s}$, making this star particularly suitable for detailed seismological analysis.

Table 1. Parameters derived for PG 1047+003 from asteroseismology.

\begin{tabular}{lcc}
\hline \hline Quantity & Asteroseismology & Spectroscopy \\
\hline $\log g$ & $5.800 \pm 0.008(0.14 \%)$ & $5.80 \pm 0.10(1.72 \%)$ \\
$T_{\text {eff }}$ & $34,000 \pm 1,900 \mathrm{~K}(5.59 \%)$ & $34,500 \pm 1000 \mathrm{~K}(2.90 \%)$ \\
$M_{\text {tot }} / M_{\odot}$ & $0.490 \pm 0.019(3.88 \%)$ & $\ldots$ \\
$\log M_{\text {env }} / M_{\text {tot }}$ & $-3.82 \pm 0.14(3.66 \%)$ & $\ldots$ \\
$M_{\text {env }} / M_{\odot}$ & $(7.4 \pm 2.7) \times 10^{-5}(36 \%)$ & $\ldots$ \\
$R / R_{\odot}\left(M_{\text {tot }}, g\right)$ & $0.146 \pm 0.005(3.42 \%)$ & $\ldots$ \\
$L / L_{\odot}\left(T_{\text {eff }}, R\right)$ & $25.7 \pm 7.5(29.2 \%)$ & $27.2 \pm 5.0(18.4 \%)^{1}$ \\
\hline \multicolumn{2}{l}{${ }^{1}$ Based on the spectroscopic value of $T_{\text {eff }}$ and the asteroseismological value of $R$}
\end{tabular}

Guided by the surface parameters of this star derived from spectroscopy (Table 1), we built a grid of 45,084 models exploring the parameter space region defined by $31,000 \mathrm{~K} \leq T_{\text {eff }} \leq 39,000 \mathrm{~K}\left(\Delta T_{\text {eff }}=500 \mathrm{~K}\right), 5.64 \leq \log g \leq$ $5.90(\Delta \log g=0.01), 0.46 \leq M_{\text {tot }} / M_{\odot} \leq 0.52\left(\Delta M_{\text {tot }} / M_{\odot}=0.10\right)-5.0 \leq$ $\log q(H) \leq-3.4(\Delta \log q(H)=0.10)$. Modes of degree $\ell=0$ up to $\ell=4$ with periods between 60 and $500 \mathrm{~s}$ were considered in the nonadiabatic pulsation computation. Looking at the resulting $4 \mathrm{D}-\chi^{2}$ function, we isolate 3 minima that possess the lowest values. However, based on the sole value of $\chi^{2}$, it appears difficult to decide which of these is the "true" solution, as all 3 minima turn out to be similar in terms of the quality of fit. Nevertheless, using additional constraints from both the nonadiabatic pulsation calculations and the independent spectroscopic values of the surface parameters, we find that among these 3 potential solutions, only one is simultaneously consistent with both the mode stability calculations (i.e., all the observed modes are assigned to modes predicted to be unstable) and the spectroscopic values of $T_{\text {eff }}$ and $\log g$.

Keeping this solution, the derived parameters are given in Table 1. These are fully consistent with the spectroscopic values. Note that $\log g$ is obtained with a much higher accuracy using asteroseismology and that quantities such as the total mass of the star and the mass of the H-rich envelope can be inferred. The corresponding simultaneous fit of the 19 periods (shown in Fig. 1) has a mean relative difference between the observed and computed periods of $\triangle P / P \sim 0.8 \%$ (the largest difference being $\Delta P / P \sim 2.17 \%$ ). We stress that inclusion of modes of degree $\ell=4$ was necessary to account for the mode density near $P \sim 135-155$ s. Indeed, we find it impossible to single out a model with relevant parameters that could account for the observed period density if we restrict ourselves to 


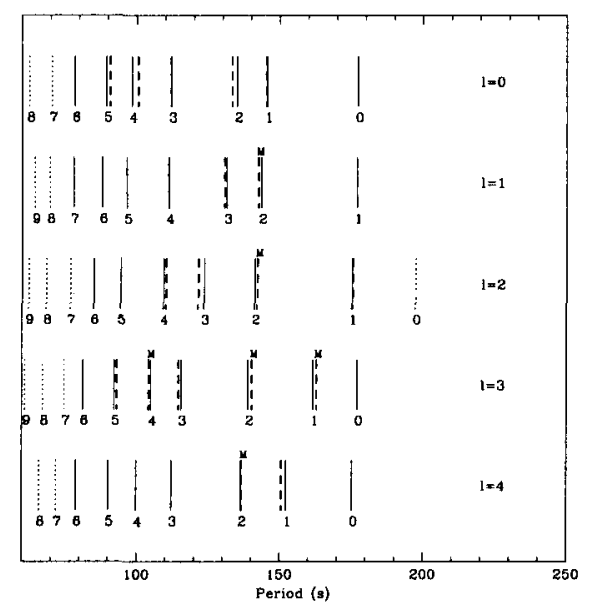

Figure 1. Period fit of the best solution found for PG 1047+003. Dashed segments are the observed periods, while plain (dotted) segments represent the computed periods of the unstable (stable) modes for $\ell=0$ up to $\ell=4$. Numbers below each segment correspond to the radial order $k$. "M" signs indicate a multiplet according to the data.

modes with $\ell \leq 3$. Hence, several modes with $\ell=4$ (two according to the fit) must be present in the period spectrum of PG $1047+003$.

These results (as well as those of Brassard et al., 2001) show that the detailed asteroseismological analysis of EC14026 stars is indeed possible and promises to be very fruitful in a near future.

\section{References}

Brassard, P. et al. 2001, ApJ, in press

Charpinet, S., Fontaine, G., Brassard, P., \& Dorman, B. 1996, ApJ, 471, L103

Charpinet, S., Fontaine, G., \& Brassard, P. 2001, PASP, 113, 175

Dorman, B., Rood, R., \& O'Connell, R. 1993, ApJ, 419, 596

Kilkenny, D., Koen, C., O'Donoghue, D., \& Stobie, R. 1997, MNRAS, 285, 640

\section{Discussion}

I. Roxburgh : You mentioned that other minima give similar quality of fit. What are they and how do they compare with the chosen solution?

$S$. Charpinet : Period fit solutions are not unique and additional constraints are needed to isolate a "preferred" solution. In this case, among the two additional local minima possible, one is in fact very close to the chosen solution (but slightly cooler and therefore less consistent with the spectroscopic temperature). The other one does not intersect with the spectroscopic $T_{\text {eff }}$ and $\log g$ uncertainty box and the period fit is not as good. 